\title{
Sources $X$ et XUV intenses et brèves : Journées d'Orsay des 5 et 6 mars 1998
}

\author{
Ph. Zeitoun, G. Petite* ${ }^{*}$ A. Klisnick, P. d'Oliveira* et G. Jamelot \\ LSAI, Université Paris-Sud, URA 775 du CNRS, bâtiment 350, 91405 Orsay cedex, France \\ * CEAIDSM/DRECAM, Saclay, 91191 Gif-sur-Yvette cedex, France
}

\begin{abstract}
Résumé: L'idée de ces journées est née du besoin de coordonner les efforts des équipes travaillant sur les nouvelles sources X et XUV intenses et brèves. Ces sources, qui arrivent maintenant à maturité, commencent à offrir des possibilités nouvelles d'applications pour de nombreux domaines de recherche. Il a paru nécessaire de faire le point des résultats obtenus récemment à la fois sur les sources et sur leurs applications, afin de lancer une prospective et de rechercher les moyens d'organiser cette communauté scientifique, harmoniser les programmes et rationaliser l'utilisation des moyens. Une question particulièrement importante et difficile a été révélée avec force au cours de ces journées. Il s'agit des besoins en optique multicouche, qui sont presque exclusivement satisfaits par des laboratoires de recherche qui ne peuvent consacrer qu'une part marginale de leur activité à cette production. En revanche, le marché est actuellement trop faible pour être pris en charge par les entreprises à un prix compatibles avec les moyens de la recherche publique.Le recueil des exposés présentés au cours de ces journées sera publié prochainement par l'Université Paris-Sud
\end{abstract}

\section{INTRODUCTION}

A la suite d'efforts soutenus, une nouvelle classe de sources XUV et $\mathrm{X}$ a connu récemment des progrès considérables et arrive à maturité. Il s'agit pour l'essentiel des lasers X produits par des plasmas amplificateurs denses et chauds, des plasmas produits par des impulsions laser de haute intensité et de la génération d'harmoniques d'ordres élevés. Il faudrait bien entendu ajouter à cette liste d'autres sources en cours de développement, en particulier les lasers à électrons libres, dont les harmoniques peuvent constituer des sources XUV intéressantes. A des degrés divers, ces sources ont pour propriétés communes forte intensité et brièveté; elles peuvent être utilisées pour des applications spécifiques à divers domaines de recherche, applications qui ne pourraient être réalisées avec les sources plus traditionnelles, tubes à rayons $X$ ou rayonnement synchrotron.

Les études de ces sources font appel à des moyens expérimentaux relativement lourds, parfois à des grands équipements. Elles nécessitent aussi le développement d'outils de simulation numérique importants. Enfin, elles impliquent l'utilisation de composants optiques, en particulier de composants interférométriques "multicouche" de très grande qualité de surface, dont la réalisation demande à la fois des connaissances de base très pointues, des moyens techniques et des "tours de main" encore peu répandus.

Enfin, le développement des sources implique la réalisation d'expériences d'application démontrant leur caractère novateur, ainsi que la poursuite du travail d'information auprès des utilisateurs potentiels, dont beaucoup ignorent encore les nouvelles possibilités ouvertes dans ce domaine.

Contrairement à la "communauté rayonnement synchrotron", nombreuse et bien organisée autour de grandes installations, les équipes qui réalisent ces nouvelles sources X-UV sont de petites équipes dispersées dans des laboratoires très divers et interagissant peu entre elles, bien qu'elles rencontrent des problèmes voisins sur le plan instrumental comme sur le plan numérique. Il a donc paru nécessaire d'organiser cette rencontre pour favoriser une interaction plus grande entre les équipes concernées et avec les utilisateurs, et tenter d'ouvrir une voie vers la constitution d'un pôle "Sources X et XUV intenses".

Le premier objectif de ces journées a été de faire, à partir de 22 exposés, une synthèse sur l'état de l'art des sources, sur les problèmes instrumentaux et numériques rencontrés, sur les techniques à développer, enfin sur les applications en cours de développement. Des tables rondes ont ensuite permis de mettre en Iumière des problèmes cruciaux dans le domaine de l'optique et de montrer à la fois la nécessité et les difficultés d'une meilleure organisation de la communauté. 
La proposition de GDR "SAXO", qui est actuellement soumise au Comité National du CNRS, a suivi de peu ces journées d'Orsay. Bien qu'élaborée tout à fait indépendamment de ces journées, cette proposition montre bien la nécessité de créer une synergie dans le domaine des sources X et XUV intenses et brèves.

\section{LES SOURCES}

Dix exposés présentés par huit laboratoires ont donné un panorama représentatif des sources intenses actuellement disponibles, ou susceptibles d'être utilisables dans un avenir proche. Ces sources peuvent être classées à partir d'un grand nombre de propriétés caractéristiques (nombre de photons par impulsion, durée des impulsions, directivité, cohérence, brillance, ...) et apparaissent clairement comme complémentaires les unes des autres. Il est important de bien cerner les propriétés de chacune de ces sources pour déterminer ensuite les plus appropriées à chaque type d'application. On peut utiliser pour cela des diagrammes du type de ceux présentés figure 1 : les sources y sont classées en fonction de
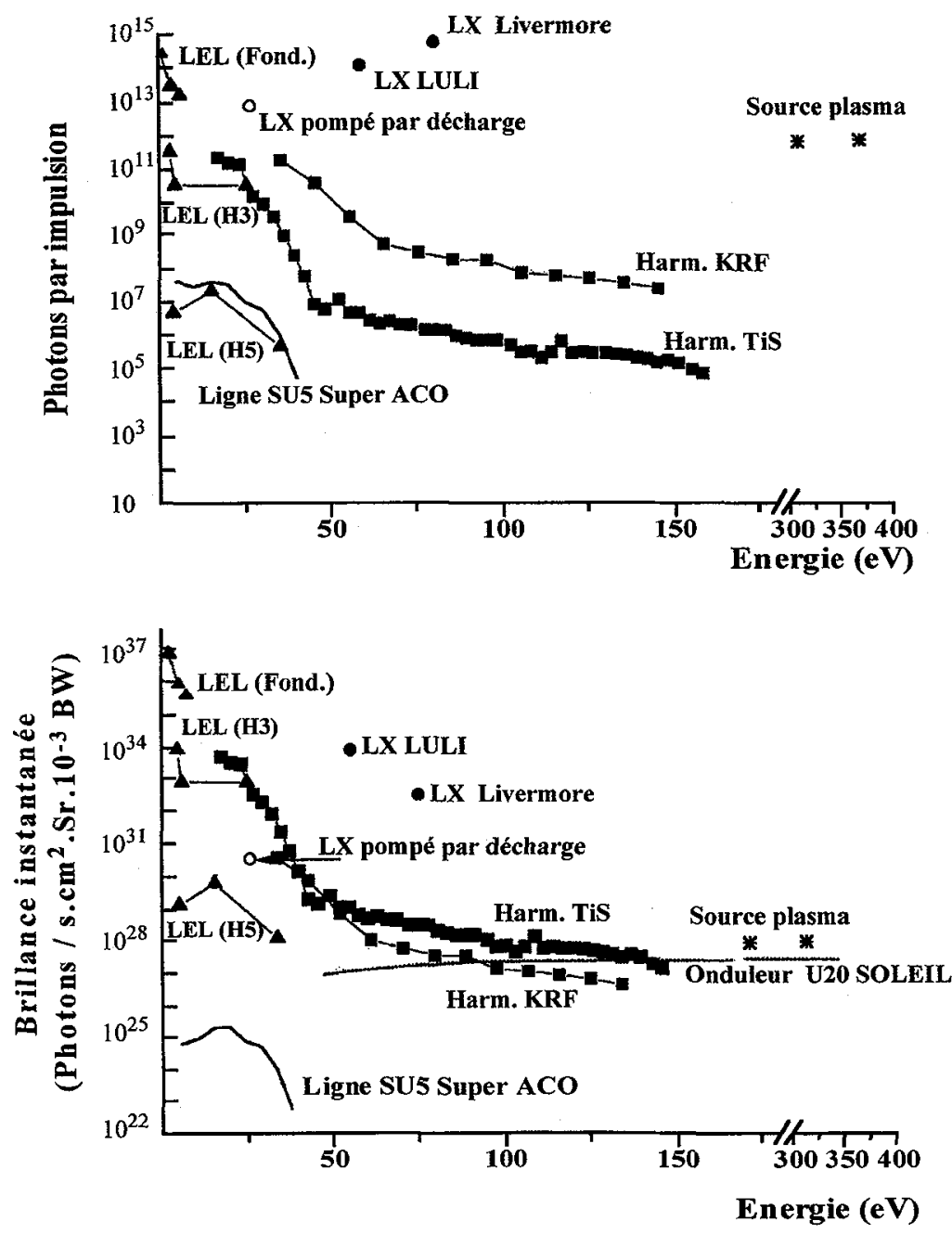

Figure 1 : Diagrammes spectraux des sources XUV actuelles en fonction du nombre total de photons par impulsions (partie superieure de la figure) et de la brillance instantanée (partie inférieure).

LEL : lasers à électrons libres. LEL (Hn) harmonique de degré na d'un LEL.

LX : lasers à rayons X du LULI, du LLNL et de l'Université de Colorado (pompé par décharge).

Harm, : harmoniques d'ordres élevés des lasers KrF et TiS.

Sources plasmas : émissions dues à l'interaction avec un laser à très courte durée d'impulsion.

Onduleur U20 : prévu dans le cadre du projet SOLEIL 
l'énergie des photons émis pour deux grandeurs caractéristiques, le nombre total de photons par impulsion et la brillance instantanée des sources.

Les sources harmoniques d'ordres élevés sont créées lors de la focalisation d'un laser visible de forte puissance dans un jet de gaz. Grâce aux progrès récents des lasers à impulsion courte, cette catégorie de sources X-UV couvre un large domaine spectral, allant de 20 à $150 \mathrm{eV}$. Ces sources ont pour caractéristiques essentielles une très courte durée d'impulsion, une bonne directivité et une cohérence élevée, directement liées aux propriétés du laser fondamental.

Les lasers de forte puissance et d'impulsion ultracourte sont également utilisés pour la production de plasmas denses qui sont des sources intenses de rayonnement $X$ continu d'origine thermique (Bremsstrahlung). Par ailleurs l'interaction laser-plasma produit des électrons rapides capables d'ioniser en couche profonde les atomes de la cible solide, qui se désexcitent en émettant le rayonnement incohérent et monochromatique des raies $\mathrm{K} \alpha$. Cette source occupe l'extrémité "grandes énergies" du spectre XUV, entre quelques centaines d'eV et quelques $\mathrm{keV}$. Elle est remarquable par sa très courte durée d'émission, de l'ordre de $100 \mathrm{fs}$, qui offre actuellement la meilleure résolution temporelle possible.

Les lasers X sont dus à l'amplification de l'émission spontanée (ASE) dans des plasmas de forme allongée. Ce sont les sources X-UV les plus brillantes actuellement disponibles grâce à la forte énergie emmagasinée (1 à $10 \mathrm{~mJ}$ ) et à la directivité de l'émission, qui dépend du rapport d'aspect (longueur / largeur) du milieu amplificateur et éventuellement de passages multiples. Les plasmas sont le plus souvent produits par la focalisation sphéro-cylindrique de lasers de puissance sur des cibles solides. Plusieurs régimes de fonctionnement, liés à la durée de l'impulsion laser (600 à $1 \mathrm{ps}$ ), peuvent être distingués. Des coefficients d'amplification très élevés ont été obtenus récemment en découplant la production de l'inversion de population (impulsion "courte") de celle du plasma (impulsion "longue"). La limitation principale de ces lasers X pour le développement d'applications est due actuellement à la très faible cadence des impulsions, limitée par la cadence de tir des lasers de forte énergie (3 tirs par heure au LULI). Des lasers pompés par diode, du type de ce qui est développé à Livermore dans le cadre du projet "Mercury" pourront permettre de surmonter ces difficultés à moyen terme

D'autres techniques de production de rayonnement X-UV sont en cours de développement. Une émission laser X quasi saturée à la longueur d'onde de $46.9 \mathrm{~nm}$ a été réalisée en pompant un gaz d'argon par une décharge électrique pulsée dans un capillaire. Mais la tentative d'obtenir une longueur d'onde plus courte avec des émetteurs plus lourds que l'argon s'est soldée jusqu'à présent par un échec. Les lasers X pompés par ionisation par effet de champ $(\mathrm{OFI})$ à l'aide d'impulsions lasers de grande puissance et de très courte durée constituent une autre classe de lasers $\mathrm{X}$. De telles émissions n'ont été obtenues jusqu'à présent que dans des expériences de démonstration qui n'ont pas encore pu être reproduites.

Des lasers à électrons libres (LEL), développés sur un grand nombre d'accélérateurs depuis la fin des années 1970, fonctionnent jusque dans le visible et l'UV proche. L'accordabilité, la cohérence temporelle et spatiale, la polarisabilité en font des sources particulièrement performantes. Leur extension vers les courtes longueurs d'onde est prévue dans les anneaux de troisième génération de type "SOLEIL".

\section{LES APPLICATIONS}

Une part notable de ces journées a été consacrée à la description d'expériences déjà réalisées ou en projet. Dans la mesure du possible, les applications ont été présentées par les utilisateurs des sources et non par ceux qui les réalisent. On a pu ainsi entrevoir les contours d'une future communauté intéressée au progrès des sources XUV intenses pour les applications. Cependant, c'est le plus souvent la proximité (thématique, géographique, etc... ) entre physiciens des sources et utilisateurs qui a été le plus souvent à l'origine des expériences. Un effort très important de prospective est à poursuivre.

On peut schématiquement distinguer deux types d'expériences, l'un pour lequel la source $\mathrm{X}$ est un instrument d'analyse non destructif (spectroscopie d'absorption, radiographie, interférométrie, diffraction...) et l'autre pour lequel elle constitue une source d'énergie capable de modifier l'état du système irradié (lithographie XUV à haute résolution, excitation et ionisation mono- ou multiphotonique, interaction rayonnement XUV - matière à haut flux...).

Les sources XUV intenses et les plasmas denses et chauds étant produits dans les mêmes laboratoires, c'est naturellement au diagnostic de plasmas qu'ont tout d'abord été utilisées les sources. La spectroscopie d'absorption et la radiographie, à l'aide de "sources plasmas" continues, sont couramment utilisées. L'interférométrie de plasmas produits par laser utilisant un laser $\mathrm{X}$ réalisée tout d'abord à Livermore avec un interféromètre Mach-Zehnder, est en cours de développement au LULI avec un interféromètre imageur de Fresnel. 
Mais l'interférométrie XUV est une technique puissante qui peut être appliquée à bien d'autres objets. Elle a permis en particulier de détecter des déformations nanométriques subies par une surface étendue sous l'effet d'un champ électrique intense.

Associée à une source $\mathrm{X}$ ultra brève, l'étude par diffraction $\mathrm{X}$ d'un film de Langmuir-Blodgett perturbé par un faisceau laser intense et bref a permis d'observer l'apparition d'un désordre dans le film bien avant la dilatation de l'échantillon par effet thermique

Les éclairements instantanés les plus élevés actuellement disponibles sont obtenus par la focalisation d'un faisceau laser XUV. Ceci permet d'étendre le domaine accessible à des études déjâ réalisées, comme par exemple celles de luminescence. L'excitation de la luminescence du CsI par laser X a ainsi permis d'observer le quenching de la luminescence à haut flux et de mettre en évidence de nouvelles bandes de luminescence, invisible lors de l'excitation par rayonnement synchrotron. D'autre part, les fortes intensités des lasers $X$ permettent de réaliser de nouvelles expériences, en particulier sur des interactions multiphotoniques.

La (nano)lithographie XUV a bien entendu de très fortes implications industrielles. La réalisation d'un ensemble comprenant une source à $14 \mathrm{~nm}$, l'optique de concentration et d'imagerie et la métrologie XUV correspondante représente un enjeu capital pour la micro-électronique. De gros efforts sont développés aux Etats Unis, mais seule une veille technologique existe en France dans ce domaine.

Une table ronde a conclu les exposés sur les sources et les applications. Elle a montré qu'il existe des convergences entre les différents laboratoires présents, des débuts de collaborations le plus souvent bilatérales. Mais elle a montré aussi les limites de ces collaborations et les difficultés pour la communauté "sources X intenses" de s'organiser, d'autant plus que les deux représentants des départements SPM et SPI du CNRS, s'ils ont pris acte des besoins exprimés au cours de ces deux jours, ont suggéré de ne pas rechercher une organisation d'ensemble. Il est probable que la proposition de GDR "SAXO", était déjà en projet à ce moment.

\section{L'OPTIQUE ET L'INSTRUMENTATION}

La qualité des composants optiques XUV est une condition nécessaire à l'utilisation efficace des propriétés des sources pour les applications. Les traitements multicouche XUV sont développés depuis les années 1970 et permettent de disposer de miroirs, mais aussi de systèmes polarisants, focalisants, imageurs de grande qualité. La situation est assez enviable actuellement pour les équipes française grâce aux efforts de laboratoires de type universitaire qui sont capables de réaliser des composants multicouche XUV de grande qualité optique.

Cependant, la table ronde "Optique" qui a suivi les exposés a montré la fragilité de cette situation et les risques de dégradation dans un avenir proche. Ces risques sont liés d'une part à des départs à la retraite non remplacés des pionniers de ce domaine, et d'autre part aux difficultés du transfert de technologie entre la recherche fondamentale, la recherche appliquée et la fabrication. Cette dernière n'entre pas en effet dans les attributions des équipes de recherche universitaires. Par contre, le "marché" des multicouches est insuffisamment développé pour pouvoir être pris en charge par l'industrie, ni du point de vue des prix, ni d'ailleurs de celui de la qualité. Une tentative américaine (OVONIX) a abouti effectivement à la réalisation de miroirs très coûteux et de qualité très inférieure à celle des miroirs réalisés au LLNL.

Cette table ronde a pu montrer avec éclat l'ampleur du problème. La recherche de solutions dépassait bien entendu ce cadre et a été confiée à un groupe de travail, coordonné par Ph. Zeitoun, qui prépare le projet d'une unité de production rattachée à l'IOTÁ.

\section{Remerciements}

Ces journées n'auraient pu être organisées sans le soutien de la Division de la Recherche de l'Université Paris-Sud et celui du CEA - DRECAM. Nous les en remercions très vivement. 